Acta Crystallographica Section D

\section{Biological Crystallography}

ISSN 0907-4449

\title{
Complex structure of type VI peptidoglycan muramidase effector and a cognate immunity protein
}

The type VI secretion system (T6SS) is a bacterial proteinexport machine that is capable of delivering virulence effectors between Gram-negative bacteria. The T6SS of Pseudomonas aeruginosa transports two lytic enzymes, Tse1 and Tse3, to degrade cell-wall peptidoglycan in the periplasm of rival bacteria that are competing for niches via amidase and muramidase activities, respectively. Two cognate immunity proteins, Tsi1 and Tsi3, are produced by the bacterium to inactivate the two antibacterial effectors, thereby protecting its siblings from self-intoxication. Recently, Tse1-Tsi1 has been structurally characterized. Here, the structure of the Tse3-Tsi3 complex is reported at $1.9 \AA$ resolution. The results reveal that Tse 3 contains a C-terminal catalytic domain that adopts a soluble lytic transglycosylase (SLT) fold in which three calcium-binding sites were surprisingly observed close to the catalytic Glu residue. The electrostatic properties of the substrate-binding groove are also distinctive from those of known structures with a similar fold. All of these features imply that a unique catalytic mechanism is utilized by Tse 3 in cleaving glycosidic bonds. Tsi3 comprises a single domain showing a $\beta$-sandwich architecture that is reminiscent of the immunoglobulin fold. Three loops of Tsi3 insert deeply into the groove of Tse 3 and completely occlude its active site, which forms the structural basis of Tse 3 inactivation. This work is the first crystallographic report describing the threedimensional structure of the Tse3-Tsi3 effector-immunity pair.

\section{Introduction}

The structural integrity of the bacterial cell is largely determined by its cell wall. Without a rigid intact cell wall, bacteria are not able to maintain cell shape, resist the internal osmotic pressure or prevent lysis (Koch, 1988). In Gram-negative bacteria peptidoglycan serves as the major structural component of the cell wall, which forms a meshwork in the periplasm surrounding the entire cell. Peptidoglycan is a singlemolecular biopolymer consisting of linear oligomeric glycan strands of alternating $\mathrm{N}$-acetylmuramic acid (MurNAc) and $\mathrm{N}$-acetylglucosamine (GlcNAc) residues cross-linked by short peptide chains of alternating L-amino and D-amino acids. Owing to its invariant structure and essential roles, the bacterial cell wall often becomes a vulnerable target for lytic enzymes, e.g. lysozyme (EC 3.2.1.17; also referred to as muramidase), effective defence molecules that exist in plants or animals to provide innate immunity against bacteria (Bugg et al., 2011; Hartl et al., 2012; Saurabh \& Sahoo, 2008).
Received 9 May 2013

Accepted 6 June 2013

PDB Reference: Tse3-Tsi3 complex, 3wa5 
Besides the immense attacking pressure from eukaryotes, bacteria also face widespread, fierce and deliberate competition for limited resources from other microbes (Hibbing et al., 2010; Brogden et al., 2005), which has shaped the evolution of a number of antagonistic pathways which commonly target bacterial cell-wall peptidoglycan (Hayes et al., 2010; Konovalova \& Søgaard-Andersen, 2011; Rendueles \& Ghigo, 2012). Specific mechanisms are required to allow cell-wall-targeting molecules to penetrate the outer membrane and to access peptidoglycan.

Many bacteria use specialized secretion systems to inject virulence factors into the target host cells. The type VI secretion system (T6SS) was recognized as a distinct class of bacterial protein secretion apparatus in 2006 (Pukatzki et al., 2006; Mougous et al., 2006). Recent studies, mostly contributed by Mougous and coworkers, have identified T6SS as a complex and widely distributed protein-export machine that is capable of cell-contact-dependent targeting of effector proteins between Gram-negative bacteria (Hood et al., 2010; Russell et al., 2011; Schwarz et al., 2010). Their pioneering work shows that the haemolysin co-regulated protein (Hcp) secretion island I-encoded T6SS of the bacterium Pseudomonas aeruginosa, a human pathogen of cystic fibrosis (CF) lung disease (Ratjen, 2009), targets at least three proteins, termed type VI secretion exported 1-3 (Tse1-Tse3), to the periplasmic space of rival bacteria (Hood et al., 2010). Among the delivered proteins, Tse 1 and Tse 3 are biochemically characterized as lytic antibacterial enzymes that degrade the peptidoglycan of recipient bacterial cells, thereby causing rapid cell lysis of competing bacteria in the environment (Russell et al., 2011). It is now known that Tse 1 functions as a peptidoglycan amidase with specificity towards $\gamma$-D-glutamyl-meso-2,6-diaminopimelic acid (D-Glu-mDAP), while Tse3 functions as a muramidase that hydrolyzes the $\beta$-1,4-linkage between MurNAc and GlcNAc. Since the peptidoglycan of $P$. aeruginosa itself is not inherently resistant to Tse 1 or Tse3, to protect its siblings the bacterium synthesizes two immunity proteins, Tsi1 and $\mathrm{Tsi} 3$, in the periplasmic compartment to specifically bind the cognate virulence effectors delivered from neighbouring cells, thereby resisting self-intoxication (Russell et al., 2011). These two effector-immunity pairs jointly confer a growth advantage to $P$. aeruginosa over its competitors.

Since the milestone work on the identification of these peptidoglycan-degrading effectors, both Tse 1 and Tse 3 have attracted intensive research from biochemists, geneticists, bioinformaticians and structural biologists. To date, crystal structures of Tse 1 alone and/or in the Tse1-Tsi1 complex have been independently determined by us (Ding et al., 2012) and in other laboratories (Shang et al., 2012; Zhang et al., 2012; Chou et al., 2012; Benz et al., 2012), and in-depth mechanical insights into the recognition and cleavage of the murein peptide substrate by Tse 1 as well as the structural basis of the Tse1Tsi1 interaction were gained from analyses of these structures. Studies of Tse 3 and Tsi3, however, are relatively incomplete, as the relevant structural information is unfortunately still unavailable. As an enzyme that cleaves the MurNAc-GlcNAc bond in the glycan backbone, Tse 3 has been functionally characterized as possessing lysozyme-like activity rather than lytic transglycosylase activity (Russell et al., 2011). In comparison with Tse1, Tse3 shows an even lower amino-acid sequence homology to known muramidases or lytic transglycosylases, despite containing a sequence motif that includes a catalytic glutamic acid found in muramidases (Russell et al., 2011). For a better understanding of the enzymatic mechanism of Tse 3 and the self-protective strategy implemented via the recognition and binding of Tse 3 by Tsi3, detailed structural information regarding this effector-immunity pair is needed.

In this study, we determined the crystal structure of the Tse3-Tsi3 complex from P. aeruginosa, which reveals unprecedented folds for both proteins. The refined structure at $1.9 \AA$ resolution also unexpectedly shows three calcium-binding sites in Tse3, one resembling the EF-hand motif and the other two being non-EF-hand sites close to the catalytic Glu residue. The crystallographic results reported here not only establish a structural framework for understanding the enzymatic mechanism underlying Tse3 lysozyme activity, but also allow us to scrutinize the structural basis for the mutual recognition and interaction between the effector and the cognate immunity protein. Moreover, the structural information presented in this article may be applicable in developing new therapeutic strategies for dealing with human diseases caused by polymicrobial infections.

\section{Experimental procedures}

\subsection{Expression and purification}

Nucleotide sequences encoding full-length Tse 3 and truncated Tsi3 without the N-terminal signal peptide (residues 1-20) were synthesized with proper optimization of the GC content (Sangon Biotech, Shanghai, People's Republic of China). Both genes were inserted into pET-22b vector (Novagen, Darmstadt, Germany) between the NdeI and XhoI endonuclease cleavage sites for cytoplasmic expression of recombinant protein with a C-terminal His tag. Protein expression in Escherichia coli strain BL21(DE3) was induced with $50 \mu M$ isopropyl $\beta$-D-1-thiogalactopyranoside when the bacterial cultures reached an $\mathrm{OD}_{600}$ of $0.6-0.8$. After $24 \mathrm{~h}$ of incubation at $289 \mathrm{~K}$, the bacterial cells were harvested by centrifugation at $4000 \mathrm{~g}$ for $30 \mathrm{~min}$.

For purification of Tse3, the cell pellets were resuspended in lysis buffer [ $150 \mathrm{~m} M \mathrm{NaCl}, 20 \mathrm{~m} M$ HEPES $\mathrm{pH}$ 7.5, $10 \mathrm{~m} M$ imidazole, $5 \mathrm{~m} M \beta$-mercaptoethanol, $5 \%(v / v)$ glycerol] with the addition of $0.1 \mathrm{~m} M$ PMSF after discarding the supernatant. Bacteria were lysed by sonication on ice at $200 \mathrm{~W}$ using $3 \mathrm{~s}$ pulses with $7 \mathrm{~s}$ intervals for $16.5 \mathrm{~min}$ before the removal of insoluble debris by centrifugation for $30 \mathrm{~min}$ at $16000 \mathrm{~g}$ and $277 \mathrm{~K}$. The recombinant protein was initially purified using $\mathrm{Ni}^{2+}$-NTA His·Bind resin (Novagen, Darmstadt, Germany) and was immediately diluted with buffer $A[50 \mathrm{~m} M \mathrm{NaCl}$, $20 \mathrm{~m} M$ HEPES pH 7.5, $1 \mathrm{~m} M$ dithiothreitol (DTT), $5 \%(v / v)$ glycerol]. The eluted fraction was further purified by cationexchange (using a $5 \mathrm{ml}$ HiTrap SP HP column, GE Healthcare, Uppsala, Sweden) and size-exclusion (using a HiLoad 16/60 
Table 1

Data-collection, phasing and refinement statistics of the Tse3-Tsi3 complex.

Values in parentheses are for the highest resolution shell.

\begin{tabular}{|c|c|}
\hline \multicolumn{2}{|l|}{ Data collection } \\
\hline Wavelength $(\AA)$ & 0.9795 \\
\hline Space group & $P 4_{3} 2_{1} 2$ \\
\hline Unit-cell parameters $\left(\AA{ }^{\circ},{ }^{\circ}\right)$ & $\begin{array}{c}a=b=109.97, c=84.95 \\
\alpha=\beta=\gamma=90\end{array}$ \\
\hline Resolution range $(\AA)$ & $49.18-1.90(2.00-1.90)$ \\
\hline Unique reflections & 41573 \\
\hline Multiplicity & $28.4(28.8)$ \\
\hline Completeness (\%) & $100.0(100.0)$ \\
\hline Mean $I / \sigma(I)$ & $28.9(11.8)$ \\
\hline Solvent content (\%) & 42.74 \\
\hline$R_{\text {merge }}$ & $0.105(0.335)$ \\
\hline \multicolumn{2}{|l|}{ SAD phasing } \\
\hline Se-atom sites & 4 \\
\hline Overall figure of merit & 0.380 \\
\hline \multicolumn{2}{|l|}{ Structure refinement } \\
\hline$R_{\text {work }}$ & 0.160 \\
\hline$R_{\text {free }}$ & 0.202 \\
\hline R.m.s.d., bond lengths $(\AA)$ & 0.007 \\
\hline R.m.s.d., bond angles $\left(^{\circ}\right)$ & 1.094 \\
\hline \multicolumn{2}{|l|}{ Ramachandran plot } \\
\hline Favoured region (\%) & 98.07 \\
\hline Allowed region (\%) & 1.74 \\
\hline Outliers (\%) & 0.19 \\
\hline
\end{tabular}

Superdex 75 column, GE Healthcare) chromatography. Selenomethionine-substituted (SeMet) Tse3 was produced in the methionine-auxotrophic E. coli strain B834 (DE3). An identical protocol to that used for the native protein was applied for the purification of the SeMet derivative, but with $10 \mathrm{~m} M \beta$-mercaptoethanol added to the lysis buffer and $5 \mathrm{~m} M$ DTT added to buffer $A$. Native Tse3 was stored in $150 \mathrm{~m} M$ $\mathrm{NaCl}, 20 \mathrm{~m} M$ HEPES pH 7.5, $1 \mathrm{~m} M$ DTT, 5\% glycerol after purification and the SeMet protein was stored in the same buffer but containing $4 \mathrm{~m} M$ DTT and $0.2 \mathrm{~m} M$ EDTA.

For purification of Tsi3, the bacterial pellets were resuspended in the same lysis buffer but without glycerol after cell harvesting. Tsi3 was purified using the same three-step procedure as Tse 3 and was stored in $150 \mathrm{mM} \mathrm{NaCl}, 20 \mathrm{~m} M$ Tris- $\mathrm{HCl} \mathrm{pH} 7.5,1 \mathrm{~m} M$ DTT. The Tse3-Tsi3 complex was obtained by mixing the two proteins with excess Tsi3 to saturate the Tse 3 and was further purified by size-exclusion chromatography using a HiLoad 16/60 Superdex 75 column (GE Healthcare, Uppsala, Sweden). The purified complex protein was stored in the same buffer as used for Tsi3 and was frozen at $193 \mathrm{~K}$ until further use.

\subsection{Crystallization and data collection}

The Tse3-Tsi3 complex was concentrated to approximately $5 \mathrm{mg} \mathrm{ml}^{-1}$ prior to crystallization trials, which were carried out at $293 \mathrm{~K}$ using the hanging-drop vapour-diffusion method. The drop in each well was formed by mixing $1 \mu$ l protein solution with $1 \mu \mathrm{l}$ screen solution and was equilibrated against $0.5 \mathrm{ml}$ reservoir solution. An initial crystallization condition was established using the Index kit from Hampton Research (Aliso Viejo, California, USA). After thorough optimization, $\mathrm{X}$-ray diffraction-quality crystals of SeMet protein were grown in $25 \%(v / v)$ Jeffamine ED-2001, $0.1 M$ bis-tris $\mathrm{pH} 6.5$.
All crystals used for X-ray data collection were transferred into cryoprotectant [reservoir solution supplemented with $10 \%(v / v)$ ethylene glycol] for approximately $10 \mathrm{~s}$ before being mounted in nylon cryoloops (Hampton Research) and flashcooled in a stream of liquid nitrogen for optimal cryoprotection. A single-wavelength anomalous diffraction (SAD) data set was collected for the SeMet-labelled Tse3-Tsi3 complex at $100 \mathrm{~K}$ at a wavelength of $0.9795 \AA$ on beamline $17 \mathrm{~A}$ of the Photon Factory, KEK, Japan using an ADSC Quantum 315r CCD detector. All X-ray diffraction data were indexed, integrated and scaled using iMosflm (Battye et al., 2011) and SCALA from the CCP4 program suite (Winn et al., 2011).

\subsection{Structure determination and refinement}

The Tse3-Tsi3 complex structure was determined by the SAD technique. Phase calculation and density modification were performed using the PHENIX program suite (Adams et al., 2010), after which a partial model was automatically built. The rest of the model was manually completed using the Coot graphics package (Emsley et al., 2010). The structure was refined using phenix.refine (Afonine et al., 2012; Headd et al., 2012) with manual modelling between refinement cycles. The final model was validated using the MolProbity server (Chen et al., 2010) and was deposited in the Protein Data Bank (PDB) as entry 3 wa5. The statistics of data collection, SAD phasing and structure refinement are given in Table 1.

\subsection{Metal-ion detection}

Three divalent metal-binding sites were clearly observed on the protein surface of Tse3, as indicated by strong spherical electron density in the $F_{\text {obs }}-F_{\text {calc }}$ map at $3.5 \sigma$. To identify the correct metal ions that should be modelled in this structure, the elemental composition was determined using inductively coupled plasma atomic emission spectroscopy (ICP-AES). For this experiment, Tse 3 alone and the Tse3-Tsi3 complex were produced separately according to the abovementioned protocol and then diluted to concentrations of 0.15 and $0.2 \mathrm{mg} \mathrm{ml}^{-1}$, respectively. The spectra of $\mathrm{Mg}^{2+}, \mathrm{Ca}^{2+}, \mathrm{Co}^{2+}$, $\mathrm{Ni}^{2+}$ and $\mathrm{Zn}^{2+}$ for the two protein samples were obtained using Vista-MPX ICP-AES (Varian, Palo Alto, California, USA), from which corresponding ion concentrations were measured.

\subsection{Structure analysis}

The final model was sent to the ProFunc server (Laskowski et al., 2005) for topological analysis, the PIC server (Tina et al., 2007) for the analysis of protein-protein interfaces and the DALI server (Holm \& Rosenström, 2010) for a structural neighbour search. Structural comparison and structure-based sequence alignment were performed using the DaliLite server (http://www.ebi.ac.uk/Tools/structure/dalilite/). All figures representing the Tse3-Tsi3 complex structure were prepared using the PyMOL molecular-visualization program (v.1.3r1; Schrödinger). 


\subsection{Mutational study and Tse3-Tsi3 kinetic assay}

In order to investigate the structural determinants of Tse3-Tsi3 recognition and binding, a series of Tsi3 mutants including R60A, S99A, E103A, Q124A and E126A were produced by site-directed mutagenesis. These mutants together with the wild-type protein were subjected to a kinetics assay at $298 \mathrm{~K}$ using a Biacore 3000 surface plasmon resonance (SPR) instrument (GE Healthcare, Uppsala, Sweden). A running buffer consisting of $50 \mathrm{~m} M$ HEPES $\mathrm{pH}$ 7.5, $100 \mathrm{~m} M \mathrm{NaCl}, 0.005 \%(v / v)$ Tween-20 was prepared, vacuum-filtered and degassed immediately prior to the experiment. The Tse3 protein dissolved in $10 \mathrm{~m} M$ sodium acetate $\mathrm{pH} 5.0$ at a concentration of $15 \mu \mathrm{g} \mathrm{ml}^{-1}$ was immobilized on six flow cells of a CM5 sensor chip with densities from 2689.1 to 3396.3 response units. The wild-type and mutant Tsi3 proteins were transferred to $150 \mathrm{~m} M \mathrm{NaCl}, 20 \mathrm{~m} M$ HEPES
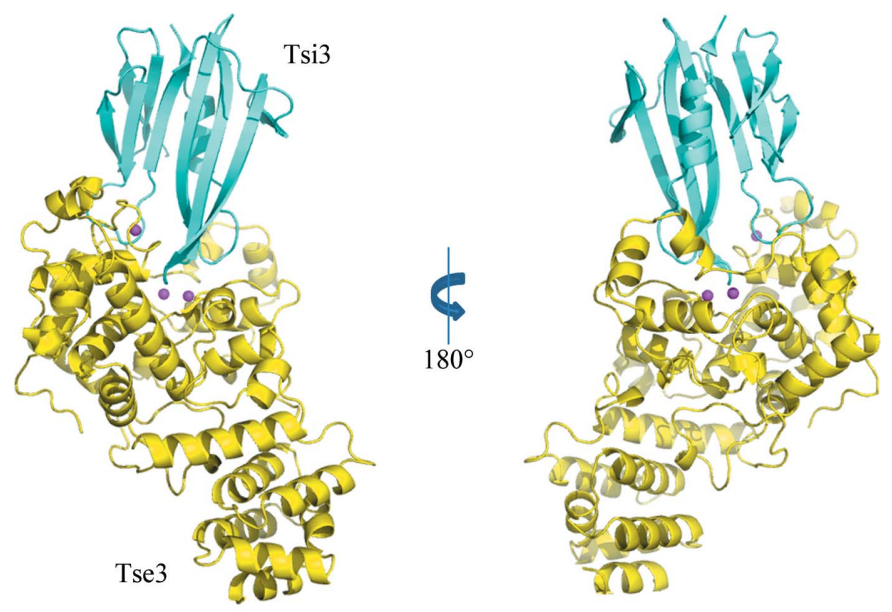

(a)

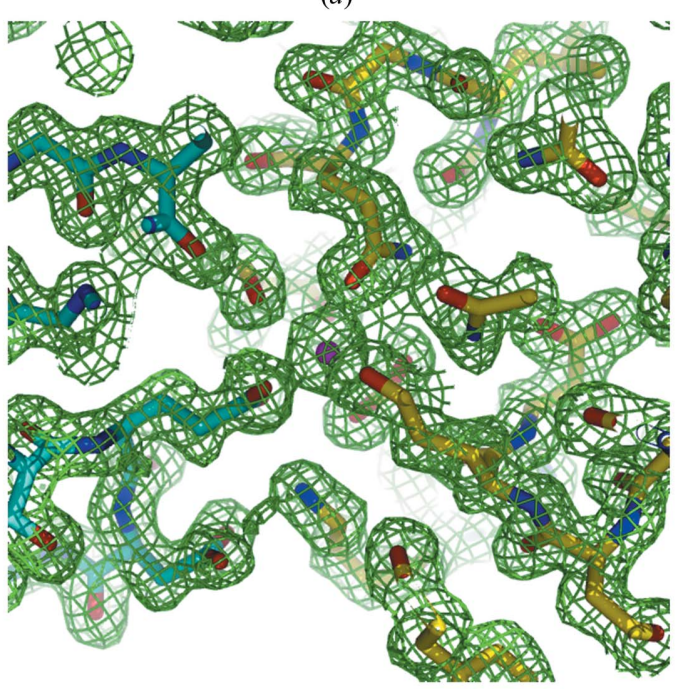

(b)

Figure 1

The overall structure and model quality of the Tse3-Tsi3 complex. (a) Ribbon diagram showing the binary complex, in which Tse 3 is coloured yellow and Tsi3 cyan. The $\mathrm{Ca}^{2+}$ ions present in Tse 3 are represented by magenta balls. $(b)$ Representative $2 F_{\text {obs }}-F_{\text {calc }}$ density map contoured at $1.0 \sigma$ of the interface between Tse 3 and Tsi3. The protein stick model is coloured by element: carbon, yellow (Tse3) or cyan (Tsi3); oxygen, red; nitrogen, blue.
pH 7.5 using three cascaded $5 \mathrm{ml}$ HiTrap desalting columns before sample injection.

Kinetic profiling was performed using the single-cycle kinetics method (Tang et al., 2006). In each analysis cycle, increasing sample concentrations were injected consecutively over the Tse 3 surfaces and a reference blank flow cell at a flow rate of $30 \mu \mathrm{min}^{-1}$. Tsi3 was diluted in the running buffer to five different concentrations in the range 0.02-1.62 $\mu M$ for the wild-type protein and the E103A and Q124A mutants and in the range 0.1-8.1 $\mu M$ for the R60A, S99A and E126A mutants. Protein samples were injected for $60 \mathrm{~s}$ at each concentration. Protein dissociation was allowed by running the buffer alone for $10 \mathrm{~min}$ after the last injection. The resultant data were analyzed using the Biacore 3000 evaluation software by fitting to a 1:1 Langmuir binding model.

\section{Results}

\subsection{Overall structure}

The crystal structure of the Tse3-Tsi3 complex was solved by SAD phasing using selenomethionine-substituted protein and was refined at $1.9 \AA$ resolution. The protein crystallized in space group $P 4_{3} 2_{1} 2$ with a single Tse 3 molecule and a single Tsi3 molecule in the asymmetric unit (Fig. 1a). The two protein molecules form a compact complex with overall dimensions of $60 \times 58 \times 93 \AA$, and the molar ratio of Tse 3 and Tsi3 agrees well with the 1:1 stoichiometry observed in solution. The final model comprises 524 amino-acid residues (400 in Tse 3 and 124 in Tsi3) and 560 heterologous atoms including three metal ions and 525 solvent molecules. Almost all protein residues are well defined in the electron-density map (Fig. 1b), except for residues 13-19 of Tse3, which did not show interpretable density in either the $2 F_{\text {obs }}-F_{\text {calc }}$ or the $F_{\text {obs }}-F_{\text {calc }}$ maps and had to be omitted from the model. The quality of the refined complex model appeared to be good in terms of both crystallographic and stereochemical parameters, which are given in Table 1.

\subsection{The structure of Tse 3}

The Tse 3 molecule displays a tilted Y-shape in the complex. In the orientation shown in Fig. 1(a), two lobes stretch from a long groove in which Tsi3 sits on Tse3. The secondary structure of Tse 3 is dominated by $\alpha$-helices, with $59 \%$ of all observed amino-acid residues distributed in a total of 28 helices, including six 310 -helices (Supplementary Fig. S1 $a^{\mathbf{1}}$ ). In sharp contrast, only one small $\beta$-sheet comprising two short antiparallel strands, $\beta 1$ (residues 274-276) and $\beta 2$ (residues 281-282), exists in the model. The $\alpha$-helices are packed tightly, which results in extensive helix-helix contacts that contribute greatly to structural integrity and stability.

Given its compact fold, it is not easy to make out domain boundaries in this model merely by eye. A structural neighbour search performed using the DALI server (Holm \&

\footnotetext{
${ }^{\mathbf{1}}$ Supplementary material has been deposited in the IUCr electronic archive (Reference: DW5056). Services for accessing this material are described at the back of the journal.
} 


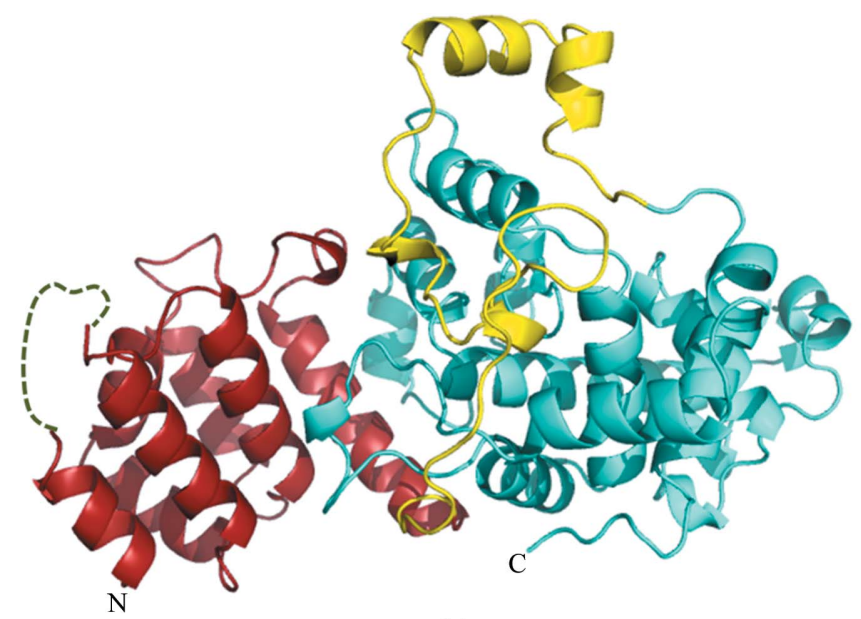

(a)

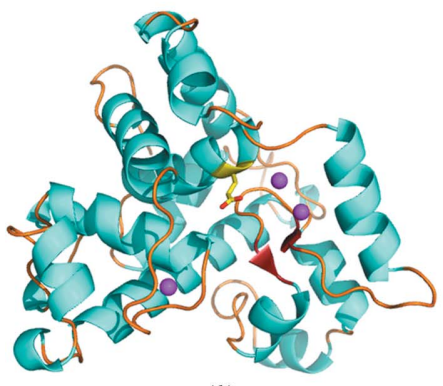

(b)

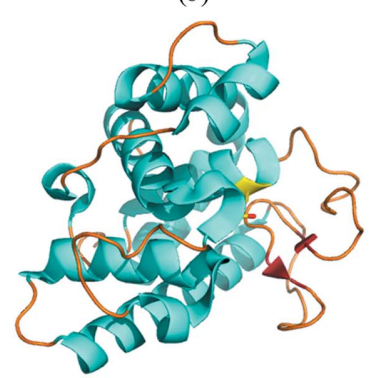

(d)

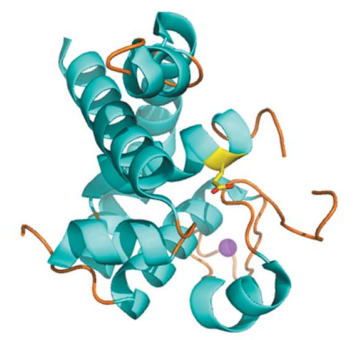

(f)

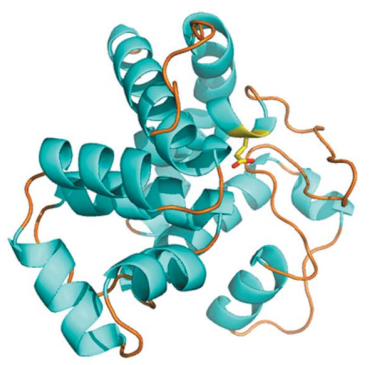

(c)

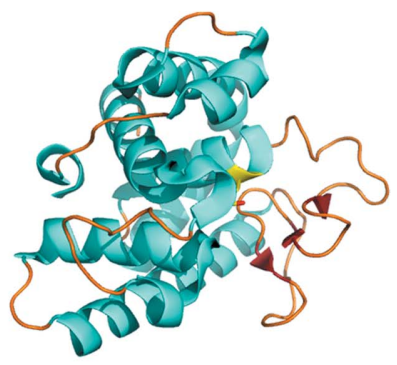

(e)

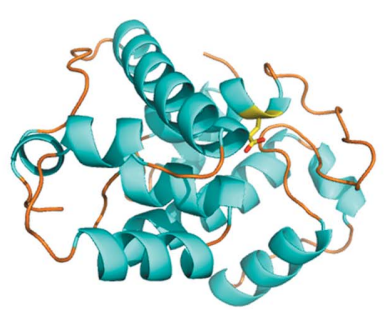

(g)

Figure 2

The structure of Tse3 and its catalytic domain in comparison with representative structures containing the SLT domain (PF01464 in the Pfam database). (a) Ribbon model of the overall structure of Tse3. The $\mathrm{N}$-terminal domain and the $\mathrm{C}$-terminal catalytic domain are coloured red and cyan, respectively, while the linker segment is shown in yellow. The loop that is not visible in the electron-density map (residues 13-19) is schematically represented by a green dashed curve. (b) Ribbon diagram of the C-terminal SLT-like domain of Tse3. $(c-g)$ Representative SLT domains of the lytic transglycosylases MltE (PDB entry 2y8p) (c), Slt35 (PDB entry 1qus) $(f)$ and Slt70 (PDB entry 1qsa) $(g)$ from $E$. coli and the goose-type lyzosymes from goose egg (PDB entry 1531) $(d)$ and Atlantic cod (PDB entry 3gxr) (e). Catalytic glutamic acids and metal ions are highlighted as stick models and magenta spheres, respectively.

Rosenström, 2010) suggests that the C-terminal peptide region from residues 183 to 408 resembles the lysozyme-like fold (SCOP 53954). The domain-organization prediction server pDomains (Veretnik et al., 2004) also recognized this peptide segment as an independent structural unit similar to the soluble lytic transglycosylase (SLT) family (Pfam PF01464). Based on these analyses, we considered decomposing the Tse3 structure into three structural modules, all of which are sequentially consecutive and make good contact with each other. Globular domains at each terminus were defined as the peptide regions from residues 2 to 126 and 183 to 408, with a linker segment in between (Fig. $2 a$ ).

Six helices $(\alpha 1, \alpha 2, \alpha 3, \alpha 5, \alpha 6$ and $\alpha 8)$ constitute an antiparallel helical bundle in the N-terminal domain (NTD), while $\alpha 5$ and $\alpha 7$ are oriented perpendicular to the bundle. Several $\beta$-turns but no $\beta$-strands are present in this domain. No structural neighbours of this domain were found by the $D A L I$ server (Holm \& Rosenström, 2010), indicating that the NTD of Tse3 represents a novel fold. The linker segment (residues 127-182) is composed of several flexible loops separated by short helices $(\alpha 9-12)$. Most of the amino acids in this module are located on the protein surface and hence show a higher degree of freedom. Two helices, $\alpha 11$ and $\alpha 12$, are involved in the formation of one lobe on the top of Tse3.

\subsection{The catalytic domain of Tse 3}

The C-terminal domain is the catalytic domain of Tse 3 as it contains Glu250, the catalytic glutamic acid identified by Russell et al. (2011). This module covers $56 \%$ of the amino acids in the primary sequence and forms the upper part of the Y-shaped molecule in the orientation shown in Fig. 1(a). It is made up of $16 \alpha$-helices from $\alpha 13$ to $\alpha 28$ and the single $\beta$-sheet present in the whole protein (Fig. $2 b$ ). A long groove is formed on top of $\alpha 17$ and the small $\beta$-sheet and is flanked by two lobes, one involving $\alpha 13$ and $\alpha 18$ and the other involving $\alpha 27$ and $\alpha 28$. As mentioned above, such an $\alpha+\beta$ structure resembles the lysozyme-like fold present in SLT domains.

The DALI server was used to search for structural neighbours of Tse3. All proteins with a $Z$-score of $\geq 8.0$ are either goose-type lysozymes or lytic transglycosylases. Representative structures of those proteins are shown in Figs. 2(c)-2(g), including the lytic transglycosylases MltE (PDB entry 2y8p; Artola-Recolons et al., 2011), Slt35 (PDB entry 1qus; van Asselt, Dijkstra et al., 1999) and Slt70 (PDB entry 1qsa; van Asselt, Thunnissen et al., 1999) from E. coli and the goose-type lysozymes from goose egg (PDB entry 1531; Weaver et al., 1995) and Atlantic cod (PDB entry 3gxr; Helland et al., 2009). All of these proteins bind peptidoglycan in a long groove containing at least six subsites, -4 to +2 , for substrate binding; the cleavage of the $\beta$-(1,4)-linkage occurs between subsites -1 and +1 . These structures share low sequence identity (11$22 \%$ ) with Tse3, but all of them can be superimposed on the catalytic domain of Tse 3 with an r.m.s.d. in the range 2.7-3.2 $\AA$ on 137-173 $\mathrm{C}^{\alpha}$ positions. Compared with its structural neighbours, Tse 3 displays two distinctive features: (i) it has a larger catalytic domain owing to several helix and loop insertions in 
Table 2

Metal-ion concentrations detected by ICP-AES.

\begin{tabular}{llllll}
\hline & \multicolumn{5}{l}{ Ion concentration $\left(\mathrm{mg} \mathrm{l}^{-1}\right)$} \\
\cline { 2 - 6 } Sample & $\mathrm{Mg}^{2+}$ & $\mathrm{Ca}^{2+}$ & $\mathrm{Ni}^{2+}$ & $\mathrm{Co}^{2+}$ & $\mathrm{Zn}^{2+}$ \\
\hline Tse3 alone & 0.050 & 0.34 & $<0.002$ & $<0.002$ & 0.003 \\
Tse3-Tsi3 & 0.046 & 0.42 & $<0.002$ & $<0.002$ & 0.018 \\
\hline
\end{tabular}

comparison with other SLT domains and (ii) it contains three metal-binding sites on the protein surface (Figs. $2 b-2 g$ ).

\subsection{Calcium-binding sites}

Calcium is a biologically important metal that commonly occurs in various eukaryotic metalloproteins, but is also observed in a few prokaryotic proteins (Yáñez et al., 2012). An example is E. coli lytic transglycosylase Slt35, which contains a noncanonical EF-hand $\mathrm{Ca}^{2+}$-binding site (van Asselt, Dijkstra et al., 1999). In this study, three metal-binding sites are surprisingly observed in the Tse 3 structure and all of them are exposed on the surface of the catalytic domain (Fig. 2b). The spherical $F_{\text {obs }}-F_{\text {calc }}$ electron density at $3.5 \sigma$ corresponding to

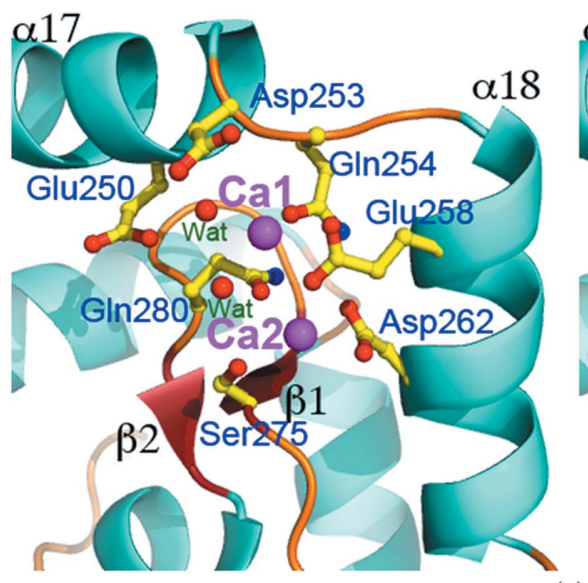

(a)
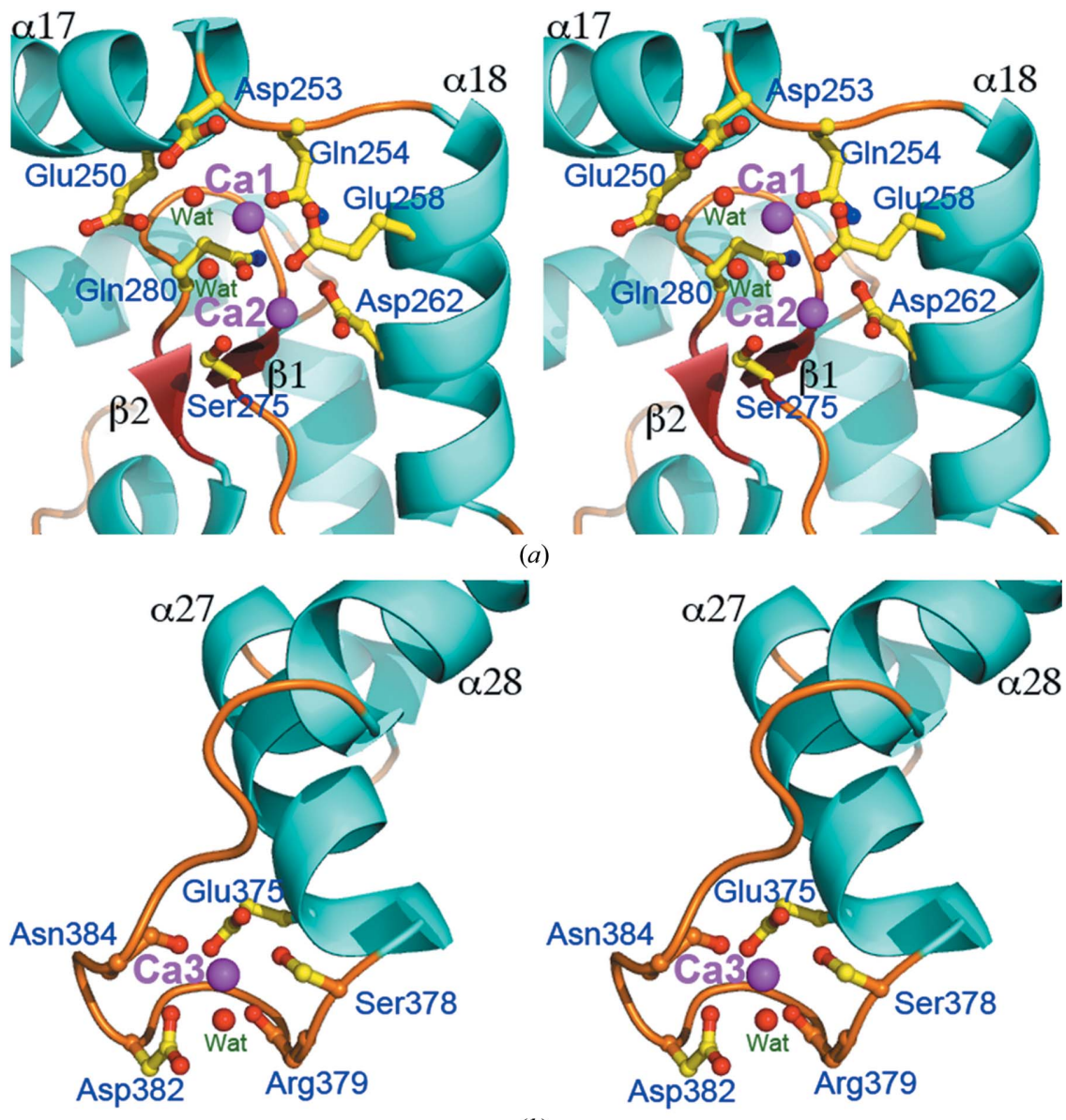

(b)

Figure 3

Stereoviews of the three $\mathrm{Ca}^{2+}$-binding sites in the structure of Tse3. (a) The adjacent non-EFhand binding sites for $\mathrm{Ca} 1$ and $\mathrm{Ca} 2$ at a position surrounded by two helices $(\alpha 17$ and $\alpha 18)$ and a two-stranded $\beta$-sheet. ( $b$ ) The EF-hand binding site for Ca3 coordinated by amino acids on a loop that connects $\alpha 27$ and $\alpha 28 . \mathrm{Ca}^{2+}$ ions are represented by magenta spheres and the ligated amino acids are shown as stick models. these sites is much stronger than that for water molecules. The sites are all coordinated by 6-7 oxygen ligands from either protein or solvent, which is a strong indicator of $\mathrm{Ca}^{2+}$ binding (Kirberger et al., 2008; Bindreither \& Lackner, 2009). To confirm this, ICP-AES was performed. The measured ion concentrations for $\mathrm{Ca}^{2+}$ were 0.34 and $0.42 \mathrm{mg} \mathrm{l}^{-1}$ for protein solutions containing Tse 3 alone and the Tse3-Tsi3 complex, respectively, which are higher than those of $\mathrm{Mg}^{2+}, \mathrm{Co}^{2+}, \mathrm{Ni}^{2+}$ and $\mathrm{Zn}^{2+}$ by one or two orders of magnitude (Table 2). The $\mathrm{Ca}^{2+}$ concentrations correspond to metal:protein molar ratios of 2.6:1 for Tse3 alone and 3.2:1 for the Tse3-Tsi3 complex, suggesting full occupancy of $\mathrm{Ca}^{2+}$ in the metal-binding sites, although no calcium salts were deliberately added to the bacterial growth medium, the buffers used in protein purification or the reservoir solutions used for crystallization. The correctness of $\mathrm{Ca}^{2+}$ modelling was indicated by perfect density fitting, comparable $B$ factors with those of protein atoms and a reasonable decrease in $R_{\text {work }} / R_{\text {free }}$ after ten cycles of restrained refinement.

All three $\mathrm{Ca}^{2+}$-binding sites are coordinated by oxygen ligands including side-chain carboxyl groups (Asp and Glu), carboxamide groups (Asn and Gln) and hydroxyl groups (Ser), as well as main-chain carbonyl O atoms and waters, with $\mathrm{Ca}-\mathrm{O}$ distances from 2.4 to $2.7 \AA$ in an overall geometry configured as pentagonal pyramidal (Fig. 3, Supplementary Fig. S2 and Supplementary Table S1). Two $\mathrm{Ca}^{2+}$ ions, named $\mathrm{Ca} 1$ and $\mathrm{Ca} 2$, are adjacently bound at non-EF-hand binding sites located midway in the groove (Fig. 2b). The protein ligands involved in these sites are located on discontinuous sequence segments, mostly from the catalytic domain, including Asp253, Gln254, Glu258 and Asp262 in the region from helix $\alpha 17$ to $\alpha 18$, and Ser275 and Gln280 in the single $\beta$-sheet (Fig. $3 a$ and Supplementary Figs. $\mathrm{S} 2 a$ and $\mathrm{S} 2 b$ ). In addition, the side chain of Asn181 from the linker segment is a ligand of Ca1. More strikingly, Glu126 of Tsi3 is involved in $\mathrm{Ca} 2$ binding, which may explain the higher occurrence of $\mathrm{Ca}^{2+}$ in the solution of the Tse3-Tsi3 complex than in that of Tse3 alone. Although Glu250 does not directly form a $\mathrm{Ca}-\mathrm{O}$ bond, it is found in the coordination shell and mediates a solvent molecule in $\mathrm{Ca} 1$ ligation. Another notable residue is $\mathrm{G} \ln 280$, the side chain of which directly ligates $\mathrm{Ca} 2$ and the mainchain carbonyl $\mathrm{O}$ atom of which directly coordinates $\mathrm{Ca} 1$ via a water molecule (Fig. $3 a$ and Supplementary Fig. S2 $a$ and $\mathrm{S} 2 b)$. The involvement of these two key catalytic residues in $\mathrm{Ca}^{2+}$ binding is astonishing and impressive, as calcium-binding sites so close to the active centre have never been observed in previously characterized 
lysozymes and lytic transglycosylases. Owing to this unique structural feature, we reason that Tse3 is very likely to exploit an unprecedented mechanism to cleave peptidoglycan substrates in which the calcium ions are involved.

The third $\mathrm{Ca}^{2+}$ ion, $\mathrm{Ca} 3$, is ligated by the side chains of Glu375, Ser378 and Asp382, as well as the main-chain $\mathrm{O}$ atoms of Arg379 and Asn384 (Fig. $3 b$ and Supplementary Fig. S2c). All of these coordinating residues are located on the loop that connects helix $\alpha 27$ to $\alpha 28$. The polypeptide chain contributing them has a conformation that resembles the EF-hand $\mathrm{Ca}^{2+}$ binding motif characterized by a helix-loop-helix structure. In contrast to the EF-hand binding site observed in Slt35, in which the central loop consists of 15 amino acids, Ca3 binds in a 12-residue loop in this structure which is consistent with the canonical EF-hand (Kirberger et al., 2008; Bindreither \& Lackner, 2009; Maki et al., 2011). Apart from the ligating

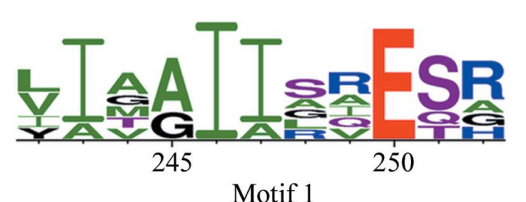

Motif 1
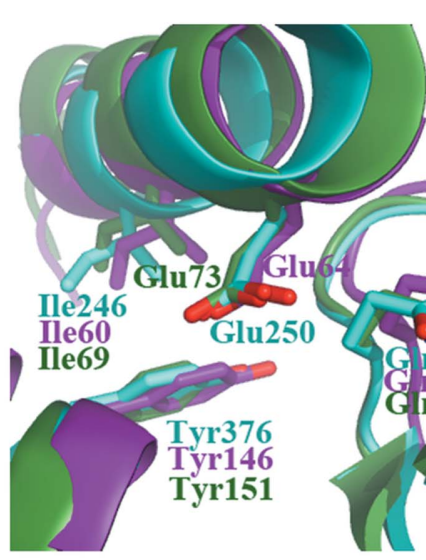

$(a)$

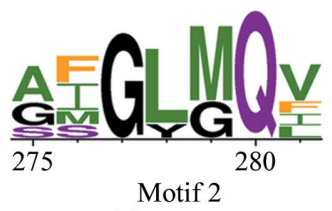

(a)
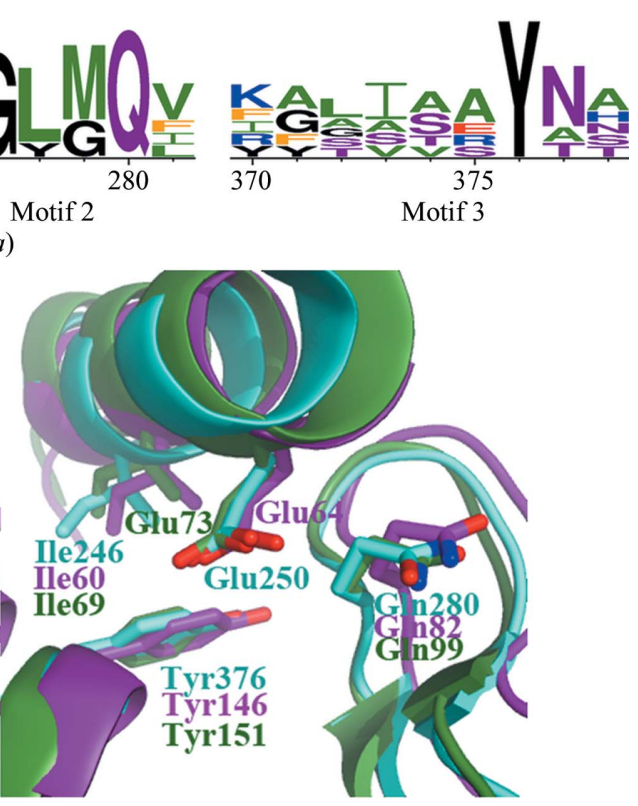

(b)

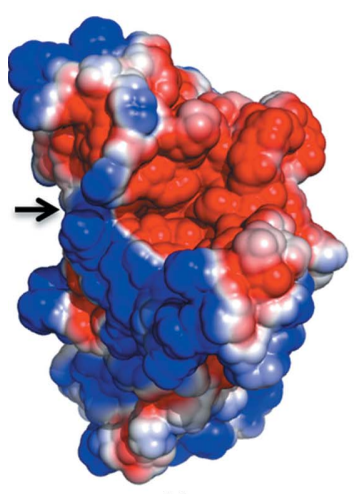

(c)

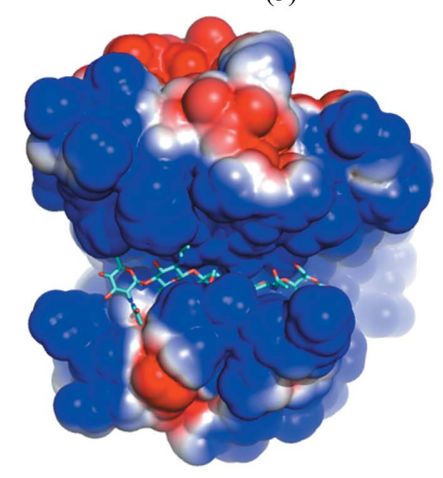

(d)

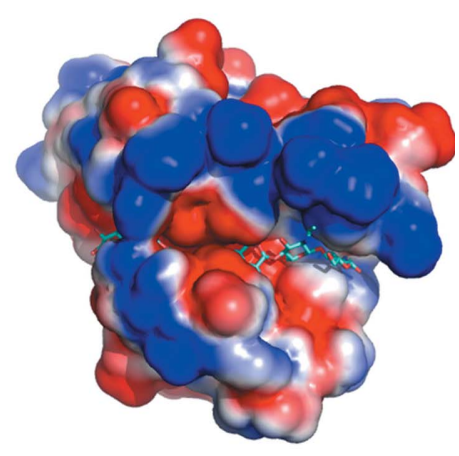

(e)
Figure 4

Sequential and structural properties of the potential active site of Tse3. (a) Three conserved motifs identified from structure-based sequence alignment between Tse 3 and other muramidases containing an SLT domain. Amino-acid conservation is depicted using WebLogo (Crooks et al., 2004). (b) Stereoview of structural superimposition of MltE (PDB entry 2y8p; purple) and gLYS (PDB entry 3gxr; green) onto Tse 3 (cyan). The invariant residues are highlighted as stick models with element colour indices. $(c)-(e)$ Electrostatic potential maps calculated on the surface of Tse 3 including the three calcium ions $(c)$, MltE $(d)$ and gLYS $(e)$. Positive and negative charges are shown in blue and red colours, respectively. The substratebinding groove in Tse 3 is indicated by a black arrow, while those in the other structures are indicated by bound glycan.
Motif 3

residues in Tse3, Glu103 of Tsi3 is also involved in Ca3 coordination via a solvent molecule. Despite its distance of from the catalytic residue, this binding site is positioned implying that $\mathrm{Ca} 3$ may also affect catalysis by probably being

\subsection{Potential substrate-binding sites}

Russell and coworkers identified Glu250 as the catalytic glutamic acid by site-directed mutagenesis (Russell et al. Tse3 using structure-prediction algorithms, which are separated by 25 amino acids. In this study, structure determination of Tse 3 allows us to perform further analyses on the basis of its structural comparison with other structurally characterized peptidoglycan-degrading enzymes containing an SLT domain. Indeed, one extra conserved motif close to the $\mathrm{C}$-terminal end was identified using secondarystructure matching (SSM) algorithms. Five positions in these three motifs are occupied by invariant amino acids, while the other positions display varying conservation among aligned sequences. The highly conserved residues are Ile246 and Glu250 in motif 1, Gly276 and Gln280 in motif 2 and Tyr376 in motif 3 (Fig. $4 a)$.

In the structure of Tse3, Ile246 is found in $\alpha 17$, Glu250 is in the loop connecting $\alpha 17$ and $\alpha 18$, and Gln280 is present in the $\beta$-hairpin bridging the two $\beta$-strands, which forms the only $\beta$-sheet in Tse3. All three residues are located at the bottom of the groove in which glycan substrates are supposed to bind. Gly276 also appears in the $\beta$-hairpin, where an amino acid without a side chain is required to form a tight turn. Tyr376 is positioned in $\alpha 27$ flanking the potential peptidoglycan-binding site. Structure superimposition shows that all of these highly conserved residues adopt identical spatial positions with almost the same side-chain rotamers (Fig. 4b), indicating their indispensable roles in enzyme activity. Apart from Glu250, which is likely to function as the catalytic acid/base in the glycosidic bond-cleavage 
reaction, Ile246, Gln280 and Tyr376 align the groove that accommodates substrates. The counterparts of Ile246 and Tyr376 in the E. coli lytic translycosylase MltE (PDB entry 4hjz; Fibriansah et al., 2012) and the goose-type lysozyme from Atlantic cod (gLYS; PDB entry 3gxr; Helland et al., 2009) form hydrophobic interactions with the sugar ring at glycan-binding subsite -2 from opposite sides, and the side chain of Gln82, the counterpart of Gln280 in MltE, forms hydrogen bonds to the $N$-acetyl and C3 hydroxyl groups on GlcNAc at subsite +1 (Fibriansah et al., 2012). All of these crystallographic data suggest that Ile246, Tyr376 and Gln280 are very likely to serve as substrate stabilizers during enzyme turnover.

The electrostatic potential map calculated on the protein surface reveals that the substrate-binding groove of Tse 3 is rich in negatively charged residues (Fig. 4c), which is another feature that is inconsistent with previously reported SLT domains that adopt a similar fold, e.g. the two structures mentioned above. In contrast to MltE (Fig. 4d) and gLYS (Fig. 4e), the grooves of which contain more positive charges than negative charges, Tse 3 is dominated by acidic residues in its groove. Apparently, this unprecedented charge distribution in Tse 3 is attributable to the abundance of glutamic and aspartic acids that coordinate the three $\mathrm{Ca}^{2+}$ ions. In other words, the occurrence of these metal ions effectively alters the electrostatic state of the catalytic groove; consequently, the substrate-binding mode in Tse 3 probably differs from its structural neighbours.

\subsection{The structure of Tsi3}

The cognate immunity protein Tsi3 sits on top of Tse 3 in the orientation shown in Fig. 1(a). It adopts an $\alpha+\beta$ architecture, differing from the all- $\beta$ fold of Tsi1 (Ding et al., 2012) and the all- $\alpha$ fold of Tsi2 (Li et al., 2012; Wang et al., 2012). Even so, Tsi 3 is built up mostly of $\beta$-strands. A total of 73 amino acids (59\%) are found in ten $\beta$-strands that form two antiparallel $\beta$-sheets, while only nine residues (7\%) form a single $\alpha$-helix. Sheet $A$ is constituted by three strands, $\beta 1, \beta 2$ and $\beta 10$, and sheet $B$ is composed of the other seven strands, $\beta 3-\beta 9$. The structure comprises two layers, one formed by sheet $A$ and the $\alpha$-helix, and the other formed by sheet $B$. Such a $\beta$-sandwich
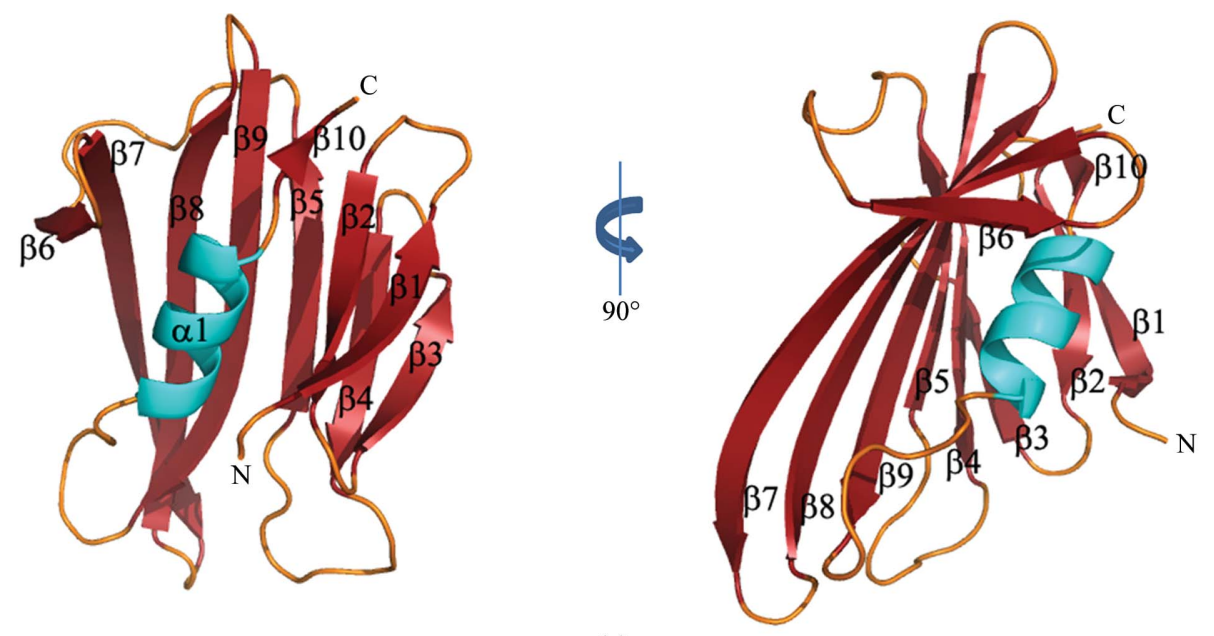

(a)

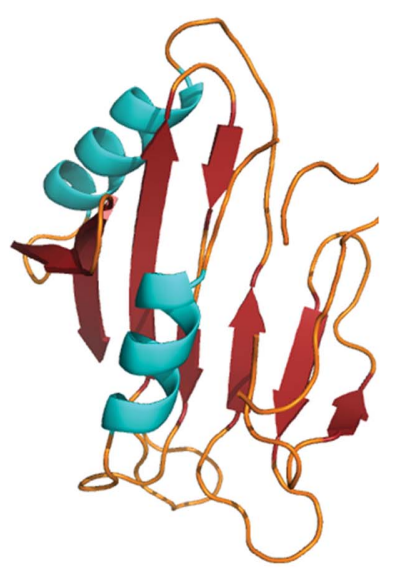

3hlz

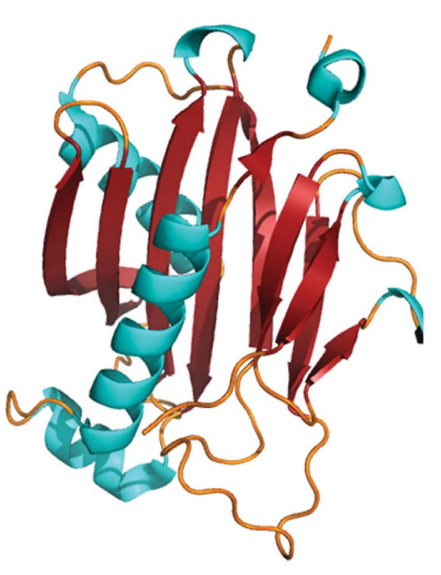

$1 e q 6$

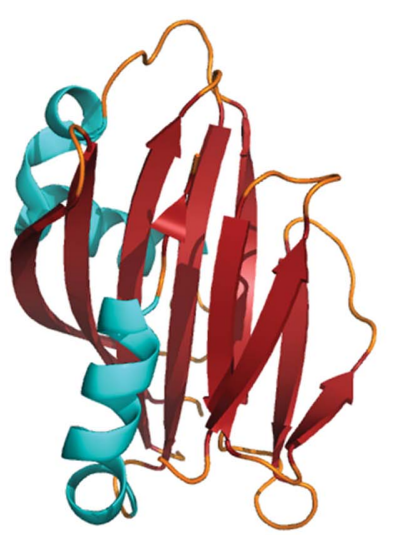

2xb3

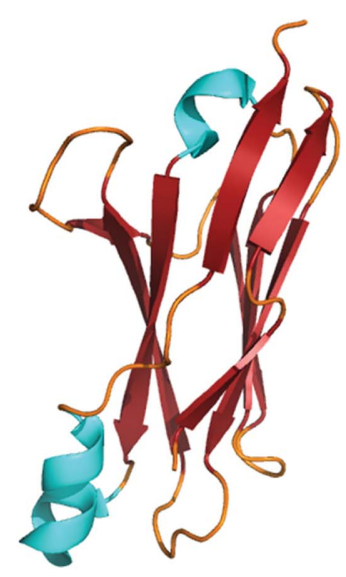

$2 \mathrm{i} 24$

Figure 5

(b)

The structure of Tsi3 and comparison with structural neighbours obtained from a DALI search (Holm \& Rosenström, 2010). (a) Ribbon representation of the structure of Tsi3; $(b)$ ribbon models of structures adopting a similar $\beta$-sandwich fold. The corresponding PDB codes are given below the structural representations. Loops, $\alpha$-helices and $\beta$-strands are coloured orange, cyan and red, respectively. 
fold can be thought of as a variant version of the jelly-roll topology with an $\alpha$-helix insertion between the two sheets.

$D A L I$ searches with Tsi3 as the input structure found that the N-terminal domain of an uncharacterized protein from Bacteroides thetaiotaomicron (PDB entry 3hlz; Joint Center for Structural Genomics, unpublished work) showed the highest structural similarity to Tsi3 (r.m.s.d. of $2.6 \AA$ on $115 \mathrm{C}^{\alpha}$ positions). Other structures with $Z$-scores above 8.0 show greater structural inconsistency with Tsi3 (r.m.s.d.s ranging from 3.4 to $4.0 \AA$ on $110-119 C^{\alpha}$ positions). A number of these structures were determined as the outcomes of structural genomic projects and have unknown functions. Those with known functions include the yeast Ran-binding protein MOG1P (PDB entry 1eq6; Stewart \& Baker, 2000) and cyanobacterial PsbP, a zinc-binding protein (PDB entry 2xb3; Michoux et al., 2010). All of the structures found by the DALI server show no sequence similarity to Tsi3 and can barely be overlaid on it, but all have a similar $\beta$-sandwich topography (Fig. 5b). Besides these structures, the structure of Tsi3 is reminiscent of the immunoglobulin fold, which is well characterized by a $\beta$-sandwich scaffold usually comprising two four-stranded sheets. More interestingly, $\alpha$-helical insertions between two $\beta$-sheet layers have been observed in some antibodies. An example is the variable domain of shark antibody IgNAR (PDB entry 2i24; Stanfield et al., 2007; Fig. 5b). All of these structures indicate that folds similar to Tsi3 occur widely in structural domains that mediate protein-protein interactions, and this is also the case for Tsi3.

\subsection{Tse3-Tsi3 interactions}

Tse 3 and Tsi3 bind tightly to each other and form a stable binary complex in the crystal. A total of $2162 \AA^{2}$ of solventaccessible surface area, with $1034 \AA^{2}$ contributed by Tse 3 (6.1\% of the total accessible surface) and $968 \AA^{2}$ contributed by Tsi3 (13.6\% of the total accessible surface), is buried upon

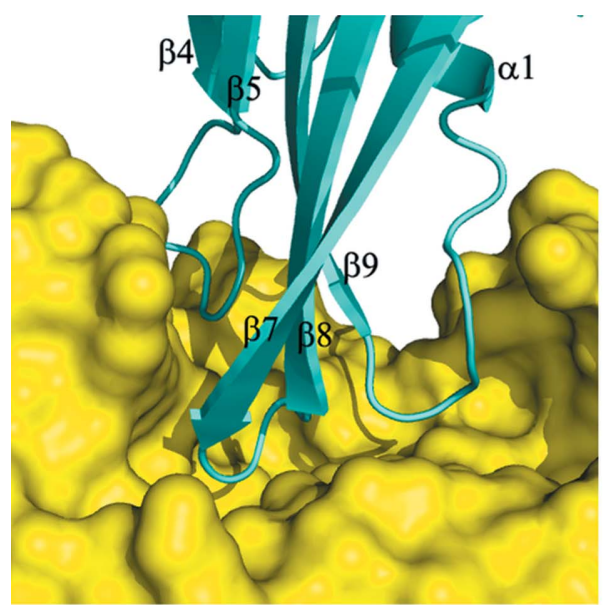

(a)

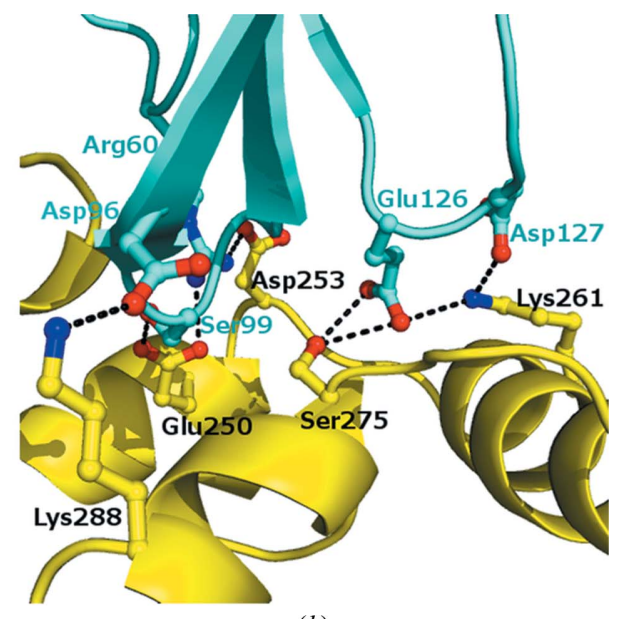

(b)
Figure 6

Tse3-Tsi3 interactions. (a) Insertion of three loops of Tsi3 (ribbon model in cyan), including two $\beta$-hairpins bridging $\beta 4-\beta 5$ and $\beta 7-\beta 8$ and a loop connecting $\beta 9$ and $\alpha 1$, into the substrate-binding groove of Tse 3 (surface representation in yellow). (b) The hydrogen-bond network formed between Tse3 (yellow) and Tsi3 (cyan) around the catalytic Glu residue in Tse3. The amino acids involved in hydrogen-bond formation are represented as stick models coloured by element. complex assembly. At the interface, 20 tight hydrogen bonds $(\leq 3.3 \AA)$ and eight charge-charge interactions $(\leq 6.0 \AA)$ are formed between the two proteins; detailed information is given in Supplementary Table 2. The formation of so many noncovalent bonds and electrostatic interactions at the protein-protein interface indicates highly mutual specific recognition of both proteins. In addition, a number of hydrophobic residues, e.g. Val176, Leu183, Val282, Trp389 and Met391 in Tse3 as well as Pro62 and Pro130 in Tsi3, are involved in forming hydrophobic interactions between the two binding components. All of these analytical data prompt us to predict strong binding between Tse 3 and Tsi3 and to hypothesize that complex assembly in this case is a spontaneous process that is probably driven by both enthalpy and entropy.

The interacting residues in Tsi3 are contributed by three loops that insert deeply into the long substrate-binding groove of Tse3 (Fig. 6a). The contacting segments include two $\beta$-hairpins bridging $\beta 4-\beta 5$ and $\beta 7-\beta 8$ and the loop connecting $\beta 9$ and the single $\alpha$-helix, all of which are rich in charged residues. The side chains of Arg60 in the first hairpin and Asp96 and Ser99 in the second hairpin are all oriented towards Glu250, the catalytic residue in Tse3, and thus form tight hydrogen bonds with this key amino acid (Fig. 6b). Coincidently, the side-chain rotamers of Arg60 and Asp96 are stabilized to a great extent by two residues carrying counter charges in the vicinity: Asp253 and Lys288 of Tse3, respectively. Consequently, a local hydrogen-bond network is formed around the catalytic centre of Tse3. Another two consecutive acidic residues, Glu126 and Asp127 in the loop following $\beta 9$ in Tsi3, form hydrogen bonds to Lys 261 on $\alpha 18$ and Ser 275 on $\beta 1$ in Tse3 through side-chain contacts (Fig. 6b), which renders the conformation of $\alpha 18$ and the small $\beta$-sheet in Tse 3 more rigid upon Tsi3 binding. As a result, the adjacent $\mathrm{Ca}^{2+}$-binding sites $(\mathrm{Ca} 1$ and $\mathrm{Ca} 2)$ harboured locally in this region are significantly stabilized, and Glu126 is directly involved in $\mathrm{Ca}^{2+}$ coordination as a ligating residue for $\mathrm{Ca}$.

The complex structure under study here straightforwardly reveals the structural mechanism by which Tsi3 effectively abolishes the enzymatic activity of Tse 3 and neutralizes its toxicity. This may occur in three ways. Firstly, Tsi3 occludes the substrate-binding sites of Tse3 by occupying the groove space (Fig. 6a). Secondly, the conformation of the active Glu residue in Tse 3 is completely fixed by the hydrogen-bond network that is formed around it (Fig. 6b). Lastly, the room for bound substrate is further decreased upon complex assembly owing to conformational changes of some structural 
Table 3

Kinetics and affinity constants for wild-type and mutant Tsi3 binding to Tse3.

\begin{tabular}{llll}
\hline Tsi3 & $\begin{array}{l}\text { Association rate } \\
k_{\mathrm{a}}\left(M^{-1} \mathrm{~s}^{-1}\right)\end{array}$ & $\begin{array}{l}\text { Dissociation rate } \\
k_{\mathrm{d}}\left(M^{-1} \mathrm{~s}^{-1}\right)\end{array}$ & $\begin{array}{l}\text { Binding affinity } \\
K_{\mathrm{d}}(M)\end{array}$ \\
\hline Wild type & $1.29 \times 10^{4}$ & $3.61 \times 10^{-4}$ & $2.81 \times 10^{-8}$ \\
R60A & Undetectable & Undetectable & - \\
S99A & $8.99 \times 10^{3}$ & $2.96 \times 10^{-2}$ & $3.29 \times 10^{-6}$ \\
E103A & $1.43 \times 10^{4}$ & $6.25 \times 10^{-4}$ & $4.36 \times 10^{-8}$ \\
Q124A & $1.73 \times 10^{4}$ & $1.01 \times 10^{-3}$ & $5.88 \times 10^{-8}$ \\
E126A & $1.21 \times 10^{4}$ & $5.05 \times 10^{-3}$ & $4.17 \times 10^{-7}$ \\
\hline
\end{tabular}

elements of Tse3. For example, the space in the binding subsite +2 is insufficient to accommodate a MurNAc residue because the loop connecting $\alpha 27$ and $\alpha 28$ becomes too close to the loop between $\alpha 12$ and $\alpha 13$ (Supplementary Fig. 3a), which is probably ascribable to the formation of hydrogen bonds and electrostatic interactions between these loops and Tsi3.

\subsection{Kinetic assay of Tse3-Tsi3 binding}

Given the extensive interaction network generated at the Tse3-Tsi3 interface, we predict high affinity between this effector-immunity pair. Consistently, in an SPR measurement performed on a Biacore 3000, wild-type Tsi3 binds immobilized Tse3 with a subnanomolar dissociation constant $\left(K_{\mathrm{d}}\right.$; Table 3 and Fig. 7). This value is lower than that for Tse1-Tsi1 by an order of magnitude, but is positively correlated with the Tse3-Tsi3 interface area of $2162 \AA^{2}$ versus that of $3951 \AA^{2}$ between Tse1 and Tsi1 (Ding et al., 2012). Still, such strong affinity is comparable with most antigen-antibody pairs. Consistent with our expectations, the Tsi3 mutants gave rise to $K_{\mathrm{d}}$ values that were increased from between twofold (E103A and Q124A) and several orders of magnitude (S99A and E126A), indicative of their relative contributions to the Tse3Tsi3 interactions. Since Ser99 and Glu126 are more greatly involved in the formation of the hydrogen-bond network at the interface than Glu103 and Gln124 (Supplementary Table 1), mutations at these positions reasonably lead to relatively weaker binding. Notably, the sensorgram of R60A showed that Tsi3 completely lost its binding activity to Tse3 upon mutation at this position (Table 3 and Fig. 7), which is suggestive of the great importance of Arg60 in assembly of the Tse3-Tsi3 complex. In fact, this result agrees well with the fact that this amino acid simultaneously forms tight salt bridges to Glu250 and Asp253 of Tse3 in this structure (Fig. $6 b$ and Supplementary Table S1) and it conclusively functions as the major structural determinant in Tse 3 recognition.

\section{Discussion}

The type VI secretion system of $P$. aeruginosa delivers three protein effectors, each of which strictly co-occurs with a cognate immunity protein. Among these effector-immunity pairs, structural studies on Tse 3 and Tsi3 have fallen behind the other two, which have been characterized using crystallographic techniques (Benz et al., 2012; Chou et al., 2012; Ding et al., 2012; Li et al., 2012; Shang et al., 2012; Zhang et al., 2012;
Wang et al., 2012). As the first crystal structure of the Tse3Tsi3 pair, the complex determined in our work provides a good opportunity to gain structural insights into the catalytic mechanism used by Tse 3 to cleave the $\beta$ - $(1,4)$-linkage in the cell-wall peptidoglycan of target bacteria and also the inhibition of its activity by the recognition and binding of Tsi3.

Metal ions present at or near the active site in enzymes often play catalytic roles rather than structural roles (Crow et al., 2009; Li et al., 2013; Luo et al., 2011; Seyedarabi et al., 2010). Three calcium-binding sites are surprisingly observed in the structure of Tse3, with only one ligated residue contributed by Tsi3. Our experimental data demonstrate the presence of $\mathrm{Ca}^{2+}$ ions at these sites. In eukaryotic proteins, calcium often serves as a key regulator in numerous biological processes, while its role in prokaryotic proteins remains elusive (Yáñez et al., 2012). The single $\mathrm{Ca}^{2+}$ site observed in the core domain of Slt35 in E. coli seems to play a more structural role, as predicted from its location $20 \AA$ away from the active site (van Asselt, Dijkstra et al., 1999). In this structure, however, all three $\mathrm{Ca}^{2+}$ ions are bound in the substrate-binding groove of Tse 3 (Figs. $3 a$ and $3 b$ ), either exposed ( $\mathrm{Ca} 1$ and $\mathrm{Ca} 2$ ) or not exposed (Ca3) to bound peptidoglycan. Their positions, in particular those of $\mathrm{Ca} 1$ and $\mathrm{Ca} 2$, are very close to the active centre, and the catalytic Glu residue even appears in the coordination shell of Ca1. This forms a striking structural feature in Tse3, as no similar sites have been observed in any other muramidases. Modelling of bound substrate by replacing $E$. coli soluble MltE by an overlaid Tse 3 catalytic domain in the structure with PDB code 4hjz (Fibriansah et al., 2012) shows that Ca1 seems to be completely accessible to the substrate at subsite +1 , and $\mathrm{Ca} 2$ and $\mathrm{Ca} 3$ are also located near the substrate within a distance of $5 \AA$ (Supplementary Fig. $3 a$ ). Even allowing for flexible substrate binding, some atoms of the substrate are inevitably positioned at hydrogen-bonding distance from certain $\mathrm{Ca}^{2+}$ ligated amino acids, in particular those coordinating Ca1.

In another aspect, the richness in Glu and Asp residues, most of which are involved in $\mathrm{Ca}^{2+}$ coordination, notably generates a unique substrate-binding groove that has abundant negative charges (Fig. 4). This distinctive electrostatic property at the active pocket of Tse 3 differs significantly from other muramidases, which contain more positive charged residues in the substrate-binding groove (van Asselt, Dijkstra et al., 1999; van Asselt, Thunnissen et al., 1999; Helland et al., 2009; Fibriansah et al., 2012). Taken together, it is reasonable to predict that the calcium ions observed in Tse 3 are very likely to play more of a catalytic role than a structural role, as in the previously reported cases in pectate lyase (Seyedarabi $e t$ al., 2010), adenylyl cyclase (Steegborn et al., 2005) and BdbD (Crow et al., 2009). They probably cause an effect in two parallel ways: (i) by direct interaction with the bound substrate via their ligated amino acids and/or (ii) by alteration of the electrostatic properties of the binding pocket. Since the involvement of metal ions in the catalysis of peptidoglycan degradation has never been reported, Tse 3 may represent a novel class of muramidase that cleaves cell-wall peptidoglycan using an unprecedented mechanism, which would probably 

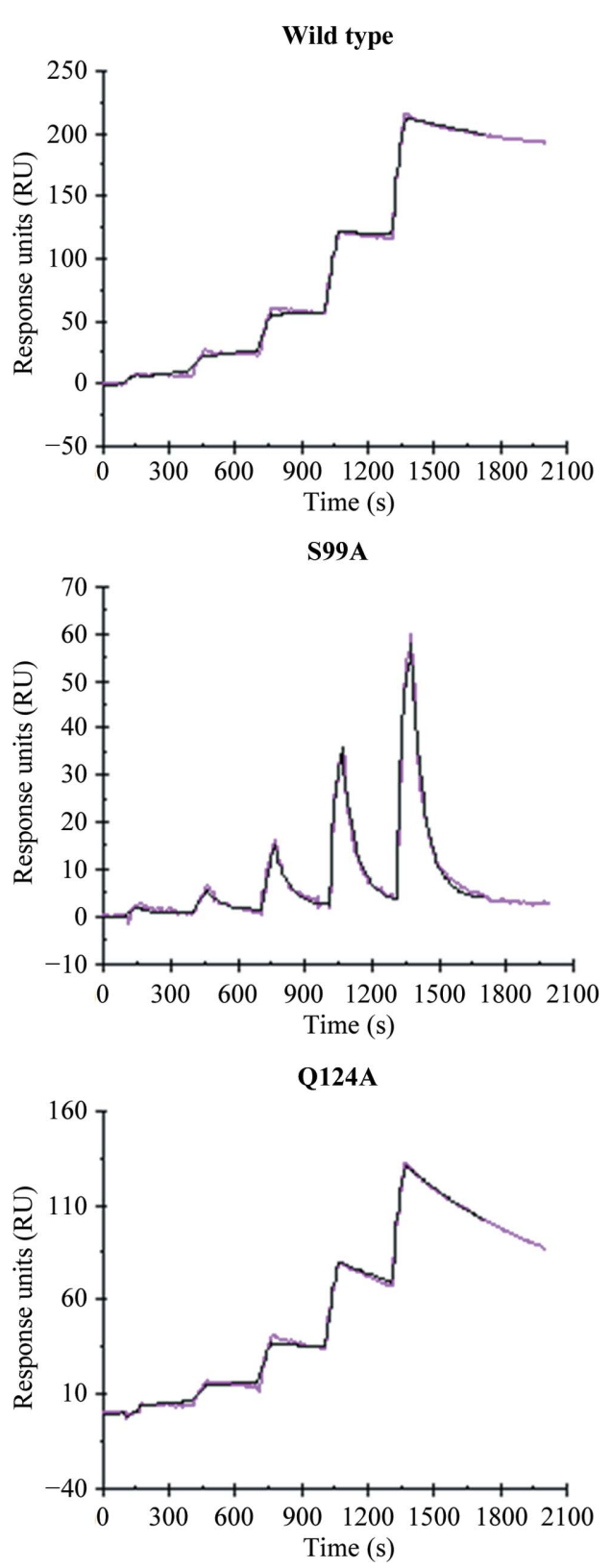

Figure 7

Sensorgrams from surface plasmon resonance experiments measuring the interactions of wild-type and mutant Tsi3 with Tse3. Raw data are represented by magenta curves, while the data fitted 1:1 to the Langmuir binding model are shown as black curves.

differ from the inverting mechanism of the goose-type lysozyme reaction, which requires a pair of catalytic acids (Hirakawa et al., 2008; Helland et al., 2009), or the anchimeric assistance mechanism of the lytic transglycosylase reaction, which relies on a single catalytic residue (Fibriansah et al., 2012).

Structural studies of Tse1 reveal that the amidase effector adopts a typical papain-like fold and shares high structural homology with the known NlpC/P60 DL-endopeptidases; hence, a canonical mechanism of murein peptide hydrolysis is expected to be linked to Tse1 (Chou et al., 2012; Ding et al., 2012). Compared with amidases, glycoside hydrolases are more common enzymes, with roles in nature that include the
R60A

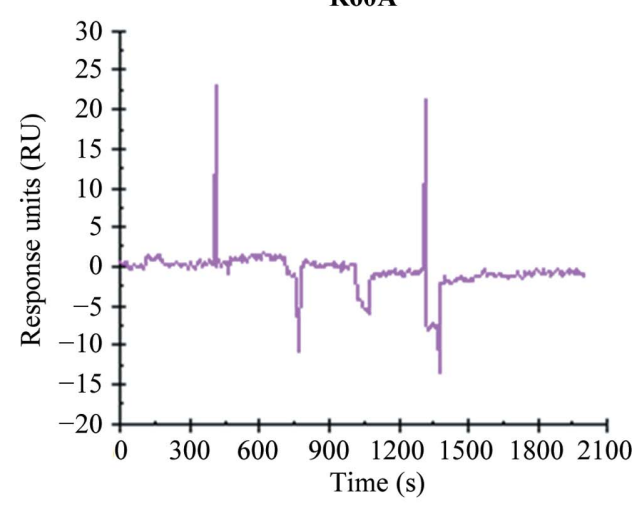

E103A

E126A

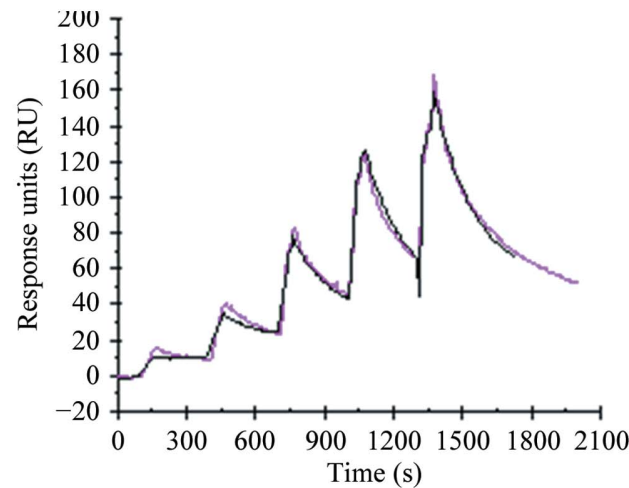
Time (s) degradation of biomass, antibacterial defence systems and cell-wall turnover (van Asselt, Dijkstra et al., 1999; van Asselt, Thunnissen et al., 1999; Fibriansah et al., 2012). On the other hand, bacteria have generally evolved antagonistic strategies to protect themselves from cell-wall degradation. In this sense, the putative unique catalytic mechanism implemented in the glycosidic bond cleavage catalyzed by Tse 3 may be significantly implicated in the competitive strategy of $P$. aeruginosa against other bacteria. One can reasonably imagine that if a peptidoglycan-cleaving effector exploiting a more general catalytic mechanism were exported from the T6SS of $P$. aeruginosa, the effector would be at risk of being inactivated by the antagonistic mechanisms existing in competing bacteria, and consequently the growth of rival bacteria cannot be effectively controlled by $P$. aeruginosa, which is certainly disadvantageous to its own growth. It follows that the bacterium may target a muramidase effector utilizing a distinctive mechanistic strategy into the periplasm of recipient Gram-negative bacteria in order to subvert their antagonistic mechanisms and thereby maximize the antibacterial efficiency. In parallel, $P$. aeruginosa utilizes a highly specific immunity protein for resisting intercellular self-intoxication. The two interrelated pathways cooperatively provide a pronounced fitness advantage for $P$. aeruginosa donor cells in the fierce niche competition.

Recently, four phylogenetically disperse families composed of peptidoglycan amidase enzymes were identified from a genomic analysis of T6SS substrates, which underscores the generality of bacteriolytic amidase effectors and cognate immunity proteins (Russell et al., 2012). Likewise, the Tse3Tsi3 muramidase effector-immunity pair is very likely not confined to $P$. aeruginosa and homologues may be identified from other Gram-negative bacteria equipped with the T6S apparatus. Further studies of these glycosidic bond-cleaving enzymes are needed in order to deepen our understanding of the underlying catalytic mechanisms, which would be of 
benefit for the rational design of antibacterial agents and the development of practical approaches to control pathogenic bacteria.

This work was supported by the '973' Project (2011CB910304, 2011CB911103 and 2013CB911500) and the National Natural Science Foundation (30725001, 31100535, 31270788 and 31270792) in China. The authors are grateful to the staff of beamline 17A at KEK, Photon Factory, Japan for data collection, and Dr Yuanyuan Chen at the Institute of Biophysics, Chinese Academy of Sciences and Dr Haijie Zou at the Center of Biomedical Analysis, Tsinghua University for their assistance in Biacore analysis.

\section{References}

Adams, P. D. et al. (2010). Acta Cryst. D66, 213-221.

Afonine, P. V., Grosse-Kunstleve, R. W., Echols, N., Headd, J. J., Moriarty, N. W., Mustyakimov, M., Terwilliger, T. C., Urzhumtsev, A., Zwart, P. H. \& Adams, P. D. (2012). Acta Cryst. D68, 352-367.

Artola-Recolons, C., Carrasco-López, C., Llarrull, L. I., Kumarasiri, M., Lastochkin, E., Martínez de Ilarduya, I., Meindl, K., Usón, I., Mobashery, S. \& Hermoso, J. A. (2011). Biochemistry, 50, 23842386.

Asselt, E. J. van, Dijkstra, A. J., Kalk, K. H., Takacs, B., Keck, W. \& Dijkstra, B. W. (1999). Structure, 7, 1167-1180.

Asselt, E. J. van, Thunnissen, A.-M. W. H. \& Dijkstra, B. W. (1999). J. Mol. Biol. 291, 877-898.

Battye, T. G. G., Kontogiannis, L., Johnson, O., Powell, H. R. \& Leslie, A. G. W. (2011). Acta Cryst. D67, 271-281.

Benz, J., Sendlmeier, C., Barends, T. R. \& Meinhart, A. (2012). PLoS One, 7, e40453.

Bindreither, D. \& Lackner, P. (2009). Gen. Physiol. Biophys. 28, F82-F88.

Brogden, K. A., Guthmiller, J. M. \& Taylor, C. E. (2005). Lancet, 365, 253-255.

Bugg, T. D., Braddick, D., Dowson, C. G. \& Roper, D. I. (2011). Trends Biotechnol. 29, 167-173.

Chen, V. B., Arendall, W. B., Headd, J. J., Keedy, D. A., Immormino, R. M., Kapral, G. J., Murray, L. W., Richardson, J. S. \& Richardson, D. C. (2010). Acta Cryst. D66, 12-21.

Chou, S., Bui, N. K., Russell, A. B., Lexa, K. W., Gardiner, T. E., LeRoux, M., Vollmer, W. \& Mougous, J. D. (2012). Cell Rep. 1, 656-664.

Crooks, G. E., Hon, G., Chandonia, J.-M. \& Brenner, S. E. (2004). Genome Res. 14, 1188-1190.

Crow, A., Lewin, A., Hecht, O., Carlsson Möller, M., Moore, G. R., Hederstedt, L. \& Le Brun, N. E. (2009). J. Biol. Chem. 284, 2371923733.

Ding, J., Wang, W., Feng, H., Zhang, Y. \& Wang, D.-C. (2012). J. Biol. Chem. 287, 26911-26920.

Emsley, P., Lohkamp, B., Scott, W. G. \& Cowtan, K. (2010). Acta Cryst. D66, 486-501.

Fibriansah, G., Gliubich, F. I. \& Thunnissen, A.-M. W. H. (2012). Biochemistry, 51, 9164-9177.

Hartl, D., Gaggar, A., Bruscia, E., Hector, A., Marcos, V., Jung, A., Greene, C., McElvaney, G., Mall, M. \& Döring, G. (2012). J. Cyst. Fibros. 11, 363-382.

Hayes, C. S., Aoki, S. K. \& Low, D. A. (2010). Annu. Rev. Genet. 44, 71-90.

Headd, J. J., Echols, N., Afonine, P. V., Grosse-Kunstleve, R. W., Chen, V. B., Moriarty, N. W., Richardson, D. C., Richardson, J. S. \& Adams, P. D. (2012). Acta Cryst. D68, 381-390.

Helland, R., Larsen, R. L., Finstad, S., Kyomuhendo, P. \& Larsen, A. N. (2009). Cell. Mol. Life Sci. 66, 2585-2598.
Hibbing, M. E., Fuqua, C., Parsek, M. R. \& Peterson, S. B. (2010). Nature Rev. Microbiol. 8, 15-25.

Hirakawa, H., Ochi, A., Kawahara, Y., Kawamura, S., Torikata, T. \& Kuhara, S. (2008). J. Biochem. 144, 753-761.

Holm, L. \& Rosenström, P. (2010). Nucleic Acids Res. 38, W545W549.

Hood, R. D. et al. (2010). Cell Host Microbe, 7, 25-37.

Kirberger, M., Wang, X., Deng, H., Yang, W., Chen, G. \& Yang, J. J. (2008). J. Biol. Inorg. Chem. 13, 1169-1181.

Koch, A. L. (1988). Microbiol. Rev. 52, 337-353.

Konovalova, A. \& Søgaard-Andersen, L. (2011). Mol. Microbiol. 81, 297-301.

Laskowski, R. A., Watson, J. D. \& Thornton, J. M. (2005). Nucleic Acids Res. 33, W89-W93.

Li, M., Le Trong, I., Carl, M. A., Larson, E. T., Chou, S., De Leon, J. A., Dove, S. L., Stenkamp, R. E. \& Mougous, J. D. (2012). PLoS Pathog. 8, e1002613.

Li, D.-F., Zhang, J.-Y., Hou, Y.-J., Liu, L., Hu, Y., Liu, S.-J., Wang, D.-C. \& Liu, W. (2013). Acta Cryst. D69, 32-43.

Luo, Y., Zhang, J., Liu, N., Luo, Y. \& Zhao, B. (2011). Life Sci. 54, 527-534.

Maki, M., Suzuki, H. \& Shibata, H. (2011). Life Sci. 54, 770-779.

Michoux, F., Takasaka, K., Boehm, M., Nixon, P. \& Murray, J. W. (2010). Biochemistry, 49, 7411-7413.

Mougous, J. D., Cuff, M. E., Raunser, S., Shen, A., Zhou, M., Gifford, C. A., Goodman, A. L., Joachimiak, G., Ordoñez, C. L., Lory, S., Walz, T., Joachimiak, A. \& Mekalanos, J. J. (2006). Science, 312, 1526-1530.

Pukatzki, S., Ma, A. T., Sturtevant, D., Krastins, B., Sarracino, D., Nelson, W. C., Heidelberg, J. F. \& Mekalanos, J. J. (2006). Proc. Natl Acad. Sci. USA, 103, 1528-1533.

Ratjen, F. A. (2009). Respir. Care, 54, 595-605.

Rendueles, O. \& Ghigo, J. M. (2012). FEMS Microbiol. Rev. 36, 972-989.

Russell, A. B., Hood, R. D., Bui, N. K., LeRoux, M., Vollmer, W. \& Mougous, J. D. (2011). Nature (London), 475, 343-347.

Russell, A. B., Singh, P., Brittnacher, M., Bui, N. K., Hood, R. D., Carl, M. A., Agnello, D. M., Schwarz, S., Goodlett, D. R., Vollmer, W. \& Mougous, J. D. (2012). Cell Host Microbe, 11, 538-549.

Saurabh, S. \& Sahoo, P. K. (2008). Aquac. Res. 39, 223-239.

Schwarz, S., Hood, R. D. \& Mougous, J. D. (2010). Trends Microbiol. 18, 531-537.

Seyedarabi, A., To, T. T., Ali, S., Hussain, S., Fries, M., Madsen, R., Clausen, M. H., Teixteira, S., Brocklehurst, K. \& Pickersgill, R. W. (2010). Biochemistry, 49, 539-546.

Stanfield, R. L., Dooley, H., Verdino, P., Flajnik, M. F. \& Wilson, I. A. (2007). J. Mol. Biol. 367, 358-372.

Shang, G., Liu, X., Lu, D., Zhang, J., Li, N., Zhu, C., Liu, S., Yu, Q., Zhao, Y., Zhang, H., Hu, J., Cang, H., Xu, S. \& Gu, L. (2012). Biochem. J. 448, 201-211.

Steegborn, C., Litvin, T. N., Levin, L. R., Buck, J. \& Wu, H. (2005). Nature Struct. Mol. Biol. 12, 32-37.

Stewart, M. \& Baker, R. P. (2000). J. Mol. Biol. 299, 213-223.

Tang, Y., Mernaugh, R. \& Zeng, X. (2006). Anal. Chem. 78, 18411848.

Tina, K. G., Bhadra, R. \& Srinivasan, N. (2007). Nucleic Acids Res. 35, W473-W476.

Veretnik, S., Bourne, P. E., Alexandrov, N. N. \& Shindyalov, I. N. (2004). J. Mol. Biol. 339, 647-678.

Wang, W., Ding, J. \& Wang, D. (2012). Prog. Biochem. Biophys. 39, 640-646.

Weaver, L. H., Grütter, M. G. \& Matthews, B. W. (1995). J. Mol. Biol. 245, 54-68.

Winn, M. D. et al. (2011). Acta Cryst. D67, 235-242.

Yáñez, M., Gil-Longo, J. \& Campos-Toimil, M. (2012). Adv. Exp. Med. Biol. 740, 461-482.

Zhang, H., Gao, Z.-Q., Su, X.-D. \& Dong, Y.-H. (2012). FEBS Lett. 586, 3193-3199. 